\title{
Equicontinuity and Sensitivity of Nondeterministic Cellular Automata
}

\author{
Pietro Di Lena \\ Department of Computer Science and Engineering \\ University of Bologna, Italy \\ pietro.dilena@unibo.it
}

\begin{abstract}
Nondeterministic Cellular Automata (NCA) are the class of multivalued functions characterized by nondeterministic block maps. We extend the notions of equicontinuity and sensitivity to multivalued functions and investigate the characteristics of equicontinuous, almost equicontinuous and sensitive NCA. The dynamical behavior of nondeterministic $\mathrm{CA}$ in these classes is much less constrained than in the deterministic setting. In particular, we show that there are transitive NCA with equicontinuous points and equicontinuous NCA that are not reversible.
\end{abstract}

Keywords: nondeterministic cellular automata, equicontinuity, sensitivity, transitivity

\section{Introduction}

Cellular Automata (CA) are discrete dynamical systems on the space of doubly infinite grid of cells. At any temporal instant, each cell can be in one of a finite number of possible states. The state of every cell is updated synchronously according to some fixed local rule that depends on the current state of the cell and that of its neighboring cells. CA represent also one of the simplest abstract models for parallel computation. The dynamical $[3,6,10,17]$ and computational $[7,9,11,12]$ properties of the CA formalism, as well as of its asynchronous and non-uniform variants $[4,5,8]$, have been well studied in literature.

Cellular Automata can be easily extended to nondeterminism by simply allowing a nondeterministic local rule. Nondeterminism is an important notion in Computation Theory, hence Nondeterministic Cellular Automata (NCA) represent a natural model for nondeterministic parallel computation. Despite its attractiveness, so far NCA received very little attention in literature. This may be due to the fact that NCA are a special class of multivalued functions and there is a substantial lack of mathematical background for studying multivalued dynamical systems [1].

The first mention of NCA in literature traces back to the seventies [20,21], and then there is a gap on the subject until very recently $[2,13-15,19]$. In [13] we started to study the most basic properties of the NCA mappings. In particular, we proved necessary and sufficient conditions that characterize the class of nondeterministic block mappings. 
In [13] we focused essentially on surjective and reversible NCA, i.e. nondeterministic block mappings whose inverse can be still defined by a nondeterministic block map. In this work we continue our investigation on NCA from the dynamical point of view. We extend some widely studied topological properties, such as equicontinuity and sensitivity, to nondeterministic mappings. These two properties do not have a standardized definition in the multivalued setting. We show several examples of equicontinuous, almost equicontinuous and sensitive NCA. In comparison to $\mathrm{CA}$, the dynamical behavior of $\mathrm{NCA}$ is much more complex and less constrained. The largest differences are probably found in the class of equicontinuous NCA. Surjective and equicontinuous CA have a strongly periodic behavior, hence they are bijective and reversible. On the contrary, there are equicontinuous NCA that are transitive and not reversible. Several questions about the NCA dynamics are open. In particular, it is an open question whether there are NCA whose set of equicontinuous points is not empty and non dense, which would imply that there are not sensitive and not almost equicontinuous NCA.

The paper is organized as follows. In Section 2 we introduce the basic notation and background. Section 3 and 4, are devoted to equicontinuity and sensitivity, respectively. In Section 5 we consider transitive NCA. Section 6 contains the final remarks.

\section{Preliminaries}

\subsection{Cellular Automata}

We introduce the basic notation and terminology we will use throughout the rest of the paper. We assume that the reader is familiar with the elementary notions from Symbolic Dynamics and Topology Theory [16, 18].

Let $A$ be a finite set with at least two elements. We denote with $A^{k}$, the set of words over $A$ of length $k>0$, with $A^{+}=\cup_{k>0} A^{k}$ the set of finite words on $A$ and with $A^{*}$ the set of finite words of $A$, including the empty word. The set $A^{\mathbb{Z}}$ denotes the set of doubly infinite sequences $\left(x_{i}\right)_{i \in \mathbb{Z}}$ of symbols $x_{i} \in A$. Given $x \in A^{\mathbb{Z}}$ we use the shortcut $x_{[i, j]}$ for the sub-word $x_{i} x_{i+1} \ldots x_{j} \in A^{j-i+1}$. A sequence containing a periodic repetition of the word $w \in A^{+}$is denoted with ${ }^{\infty} w^{\infty}$, i.e $x={ }^{\infty} w^{\infty}$ if $\forall i \in \mathbb{Z}, x_{[i \cdot|w|,(i+1)|w|-1]}=w$.

The mapping $\sigma: A^{\mathbb{Z}} \rightarrow A^{\mathbb{Z}}$, defined by $\sigma(x)_{i}=x_{i+1}$, is called shift map. The pair $\left(A^{\mathbb{Z}}, \sigma\right)$ is a dynamical system, called full shift.

Consider the metric $d(x, y)=2^{-n}$ on $A^{\mathbb{Z}}$, where $n=\min \left\{|i| \mid x_{i} \neq y_{i}\right\}$. The full shift $A^{\mathbb{Z}}$ endowed with metric $d$ is a Cantor space, i.e. a compact, totally disconnected, metric space. For every word $u \in A^{+}$and $i \in \mathbb{Z}$, the set $[u]_{i}=\left\{x \in A^{\mathbb{Z}} \mid x_{[i,|u|-1]}=u\right\}$ is called cylinder set. A cylinder set is a clopen (closed and open) set in $A^{\mathbb{Z}}$. Given $x \in A^{\mathbb{Z}}$ and $\epsilon=2^{-r}>0$, the open ball $\mathcal{B}_{\epsilon}(x)=\left\{y \in A^{\mathbb{Z}} \mid d(x, y)<\epsilon\right\}$ coincides with the cylinder set $\left[x_{[-r, r]}\right]_{-r}$. Every open set $U \subseteq A^{\mathbb{Z}}$ is defined by a countable union of cylinders. 
An endomorphism $F: A^{\mathbb{Z}} \rightarrow A^{\mathbb{Z}}$ is a sliding block code if there exists a block map $f: A^{2 r+1} \rightarrow A$, for some radius $r \geq 0$, such that for every point $x \in A^{\mathbb{Z}}, F(x)_{i}=f\left(x_{[i-r, i+r]}\right)$. We call $f$ local rule of $F$. The fundamental Theorem of symbolic dynamics [16], states that a mapping $F: A^{\mathbb{Z}} \rightarrow A^{\mathbb{Z}}$ is a sliding block code if and only if $F$ is continuous and commutes with the shift, i.e. $F(\sigma(x))=\sigma(F(x))$. The shift map $\sigma$ itself is a sliding block code. The continuous and $\sigma$-commuting endomorphisms of the full shift $\left(A^{\mathbb{Z}}, F\right)$ are usually known as Cellular Automata (CA).

\subsection{Nondeterministic Cellular Automata}

Nondeterministic Cellular Automata (NCA) are the class of multivalued functions (or multimaps) definable by nondeterministic block maps.

Definition 1. Let $A$ be some alphabet $A$ with at least two elements.

- A mutivalued mapping $f: A^{2 r+1} \rightrightarrows A$ of radius $r \geq 0$ is a nondeterministic block map if,

$$
\forall w \in A^{2 r+1}, f(w) \subseteq A
$$

- A multivalued function $F: A^{\mathbb{Z}} \rightrightarrows A^{\mathbb{Z}}$ is a nondeterministic cellular automaton if there is some nondeterministic block map $f: A^{2 r+1} \rightrightarrows A$ such that:

$$
\forall x \in A^{\mathbb{Z}}, F(x)=\left\{y \in A^{\mathbb{Z}} \mid \forall i \in \mathbb{Z}, y_{i} \in f\left(x_{[i-r, i+r]}\right)\right\}
$$

Continuity notion for (single-valued) functions can be extended to multivalued functions by means of the dual concepts of upper and lower semicontinuity (also referred to as upper and lower hemicontinuity), which collapse to the ordinary notion of continuity in the single-valued setting. The upper and lower semicontinuity properties have a simple characterization in terms of preimages of closed and open sets.

Definition 2. Let $F: A^{\mathbb{Z}} \rightrightarrows A^{\mathbb{Z}}$ be a multimap.

$-F$ is said upper semicontinuous at $x \in A^{\mathbb{Z}}$ if for any open subset $V \subseteq A^{\mathbb{Z}}$ such that $F(x) \subseteq V$,

$$
\exists \delta>0 \text { such that } \forall x^{\prime} \in \mathcal{B}_{\delta}(x), F\left(x^{\prime}\right) \subseteq V
$$

- $F$ is said lower semicontinuous at $x \in A^{\mathbb{Z}}$ if for any open subset $V \subseteq A^{\mathbb{Z}}$ such that $F(x) \cap V \neq \emptyset$,

$$
\exists \delta>0 \text { such that } \forall x^{\prime} \in \mathcal{B}_{\delta}(x), F\left(x^{\prime}\right) \cap V \neq \emptyset
$$

- $F$ is said continuous if it is both lower and upper semicontinuous at every $x \in A^{\mathbb{Z}}$.

Proposition 1. Let $F: A^{\mathbb{Z}} \rightrightarrows A^{\mathbb{Z}}$ be a multimap. 
1. $F$ is upper semicontinuous if and only if for any closed set $V \subseteq A^{\mathbb{Z}}, F^{-1}(V)$ is closed in $A^{\mathbb{Z}}$.

2. $F$ is lower semicontinuous if and only if for any open set $V \subseteq A^{\mathbb{Z}}, F^{-1}(V)$ is open in $A^{\mathbb{Z}}$.

It is easy to prove that nondeterministic block mappings are $\sigma$-commuting and continuous. However, these two properties alone are not sufficient to characterize the class of multi-valued functions definable by nondeterministic block maps.

Definition 3. Let $F: A^{\mathbb{Z}} \rightrightarrows A^{\mathbb{Z}}$ be a multimap.

- We say that $F$ is locally independent at $x \in A^{\mathbb{Z}}$ if

$$
y \notin F(x) \text { if and only if } \exists i \in \mathbb{Z} \text { such that } y_{i} \notin F(x)_{i}
$$

where

$$
F(x)_{i}=\left\{a \in A \mid \exists z \in F(x), z_{i}=a\right\}
$$

- We say that $F$ is locally independent if it is locally independent at every $x \in A^{\mathbb{Z}}$.

Theorem 1. [13] A multimap $F: A^{\mathbb{Z}} \rightrightarrows A^{\mathbb{Z}}$ is a NCA if and only if it is continuous, $\sigma$-commuting and locally independent.

It is not generally true for multivalued functions that the continuous image of a compact set is compact. It is possible to prove that this property holds for nondeterministic block mappings.

Theorem 2. [13] Let $\left(A^{\mathbb{Z}}, F\right)$ be a NCA. Then $F(U)$ is compact for every compact subset $U \subseteq A^{\mathbb{Z}}$.

An interesting class of NCA is the class of reversible NCA.

Definition 4. Let $\left(A^{\mathbb{Z}}, F\right)$ be a $N C A$.

- The reversed map $F^{-1}: F\left(A^{\mathbb{Z}}\right) \rightrightarrows A^{\mathbb{Z}}$ is defined by

$$
F^{-1}(x)=\left\{y \in A^{\mathbb{Z}} \mid x \in F(y)\right\}
$$

- We say that $F$ is a reversible NCA if $F^{-1}$ is defined by a nondeterministic block map.

In the deterministic setting reversibility coincides with the injectivity property. In the nondeterministic setting, the scenario is more complex.

Definition 5. Let $\left(A^{\mathbb{Z}}, F\right)$ be a $N C A$. We say that $F$ is injective if

$$
\forall x, y \in A^{\mathbb{Z}}, x \neq y, F(x) \cap F(y)=\emptyset .
$$




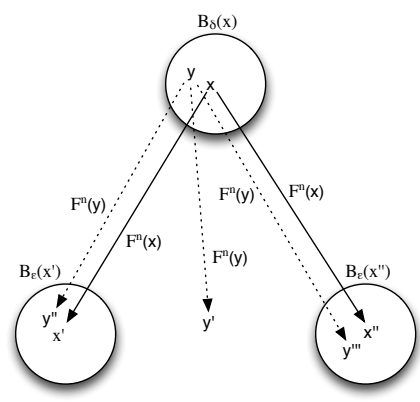

Fig. 1. lower equicontinuous point - upper sensitive

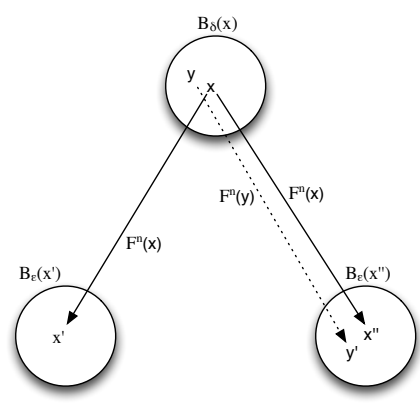

Fig. 2. upper equicontinuous point - lower sensitive

In [13] we showed that there are no injective NCA other than the class of injective CA. Moreover, if a CA $\left(A^{\mathbb{Z}}, F\right)$ is reversible, then $\left(A^{\mathbb{Z}}, F^{-1}\right)$ is an injective $\mathrm{CA}$, which implies that if $\left(A^{\mathbb{Z}}, F\right)$ is strictly nondeterministic, surjective and reversible, then $\left(A^{\mathbb{Z}}, F^{-1}\right)$ is again strictly nondeterministic. A further characteristic of reversible NCA is that they don't need to be surjective. The simplest non trivial examples of (surjective or not surjective) reversible NCA are the class of NCA with radius zero.

\section{Equicontinuity}

Equicontinuous dynamical systems are characterized by the presence of stable points, called equicontinuity points, under the iterations of the map. Dynamical systems whose set of equicontinuity points is dense (residual), are called almost equicontinuous. In the CA dynamical systems, the set of equicontinuity points is either dense or empty, and it is inversely invariant. We show an example of NCA whose set of equicontinuous points is not inversely invariant, while it is an open question whether there are NCA that have a non empty and non dense set of equicontinuous points. Furthermore, it is well known that surjective equicontinuous CA are injective, hence bijective. We show an example of surjective, equicontinuous NCA that is not reversible.

A point $x$ is called equicontinuous if the family of iterations $\left(F^{n}\right)_{n \geq 0}$ is equicontinuous at $x$. As for the continuity notion for multivalued functions, the dual properties of lower equicontinuity and upper equicontinuity facilitate the extension of equicontinuity to iterations of the multimap.

Definition 6. Let $F: A^{\mathbb{Z}} \rightrightarrows A^{\mathbb{Z}}$ be a $N C A$.

- We say that $x \in A^{\mathbb{Z}}$ is an upper equicontinuous point (Fig. 2) if

$$
\forall \epsilon>0, \exists \delta>0 \text { such that } \forall y \in B_{\delta}(x), \forall n \geq 0, F^{n}(y) \subseteq B_{\epsilon}\left(F^{n}(x)\right)
$$


- We say that $x \in A^{\mathbb{Z}}$ is a lower equicontinuous point (Fig. 1) if

$$
\forall \epsilon>0, \exists \delta>0 \text { such that } \forall y \in B_{\delta}(x), \forall n \geq 0, F^{n}(x) \subseteq B_{\epsilon}\left(F^{n}(y)\right)
$$

- We say that $x \in A^{\mathbb{Z}}$ is an equicontinuous point if it is both upper and lower equicontinuous:

$$
\forall \epsilon>0, \exists \delta>0 \text { such that } \forall y \in B_{\delta}(x), \forall n \geq 0, B_{\epsilon}\left(F^{n}(x)\right)=B_{\epsilon}\left(F^{n}(y)\right)
$$

Definition 7. Let $\mathcal{E} \subseteq A^{\mathbb{Z}}$ be the set of equicontinuous points of $\left(A^{\mathbb{Z}}, F\right)$.

- We say that $\left(A^{\mathbb{Z}}, F\right)$ is equicontinuous if $\mathcal{E}=A^{\mathbb{Z}}$.

- We say that $\left(A^{\mathbb{Z}}, F\right)$ is almost equicontinuous if $\mathcal{E}$ is a residual set.

The most simple class of equicontinuous CA is the class of mappings defined by local rules of radius zero. Such class can be easily characterized also for NCA.

Proposition 2. Any NCA with radius zero is equicontinuous.

Proof. If the local rule $f$ has radius zero, we have that $\forall x \in A^{\mathbb{Z}}, \forall n \geq 0$, $F^{n}(x)_{i}=f^{n}\left(x_{i}\right)$. This implies that $\forall \epsilon=2^{-k}>0$, if $y \in B_{\epsilon}(x)=\left[x_{[-k, k]}\right]_{-k}$ then $\forall n \geq 0$

$$
\begin{aligned}
B_{\epsilon}\left(F^{n}(x)\right) & =\left[F^{n}(x)_{[-k, k]}\right]_{-k}=\left[f^{n}\left(x_{[-k, k]}\right)\right]_{-k}=\left[f^{n}\left(y_{[-k, k]}\right)\right]_{-k}= \\
& =\left[F^{n}(y)_{[-k, k]}\right]_{-k}=B_{\epsilon}\left(F^{n}(y)\right)
\end{aligned}
$$

It is well known that every surjective equicontinuous CA is injective, hence reversible. We have already shown in [13] that every NCA with radius zero, surjective or not, is reversible. Thus nondeterministic local rules or radius zero give rise to a non trivial class of (either surjective or not) equicontinuous and reversible NCA. However, we can easily show that not every surjective and equicontinuous NCA is reversible.

Example 1. (Irreversible and equicontinuous NCA) Consider the NCA $\left(A^{\mathbb{Z}}, F\right)$ on the alphabet $A=\{0,1\}$, defined by the following local rule:

$$
\forall a, b, c \in A, f(a, b, c)= \begin{cases}\{0,1\} & \text { if } a=1, b=0, c=1 \\ \{b\} & \text { otherwise }\end{cases}
$$

The mapping $F$ is essentially the identity on $A$, except for the word 101, which is mapped nondeterministically by the local rule to $\{0,1\}$. $F$ is clearly surjective, since $\forall x \in A^{\mathbb{Z}}, x \in F(x)$. In order to see that $\left(A^{\mathbb{Z}}, F\right)$ is equicontinuous, note that 1 is a quiescent symbol, i.e. $\forall x \in A^{\mathbb{Z}}, \forall n \geq 0$ if $x_{i}=1$ then $F^{n}(x)_{i}=\{1\}$. The symbol 0 is quiescent everywhere except when it is immediately surrounded by two (quiescent) $1 s$. Then,

$$
\forall w \in A^{3}, \forall x, y \in[w]_{-1}, \forall n \geq 0, F^{n}(x)_{0}=F^{n}(y)_{0}
$$


and, generalizing,

$$
\forall w \in A^{2 k+1}, \forall x, y \in[w]_{-1}, \forall n \geq 0, F^{n}(x)_{[-k+1, k-1]}=F^{n}(y)_{[-k+1, k-1]}
$$

which implies equicontinuity. We conclude by showing that $F$ is not reversible. Consider the configuration $\tilde{x}={ }^{\infty} 1^{\infty}$, and note that $\tilde{x} \in F\left({ }^{\infty}(01)^{\infty}\right)$ and $\tilde{x} \notin F\left({ }^{\infty} 0^{\infty}\right)$. Now, if $F^{-1}$ is defined by some nondeterministic block map $f^{-1}: A^{2 k+1} \rightrightarrows A$, the only possibility is that $f^{-1}\left(1^{2 k+1}\right)=\{0,1\}$. But in this way, $F^{-1}(\tilde{x})=A^{\mathbb{Z}}$, while ${ }^{\infty} 0^{\infty} \notin F^{-1}(\tilde{x})$.

In topological (single-valued) dynamical systems, the set of equicontinuous points is inversely invariant. This property does not hold for multivalued mappings. The following example shows an almost equicontinuous NCA whose set of equicontinuous points is not invariant.

Example 2. (Almost equicontinuous NCA with not inversely invariant equicontinuous points) Consider the $\operatorname{NCA}\left(A^{\mathbb{Z}}, F\right)$ on alphabet $A=\{0,1,2\}$, defined by the following nondeterministic local rule:

$$
\forall a, b, c \in A, f(a, b, c)= \begin{cases}\{2\} & \text { if } a=2 \text { or } b=2 \text { or } c=2 \\ \{0,2\} & \text { if } a=b=c=0 \\ \{c\} & \text { otherwise }\end{cases}
$$

Note that, the symbol 2 is a quiescent symbol that spreads to the left and to the right. The point ${ }^{\infty} 2^{\infty}$ is thus an equicontinuous point of $\left(A^{\mathbb{Z}}, F\right)$. Consider the set of sequences that contain infinitely many occurrences of the symbol 2 to the left and to the right.

$$
U=\left\{x \in A^{\mathbb{Z}} \mid \forall i \in \mathbb{N}, \exists k^{\prime} \geq i, k^{\prime \prime} \leq-i, \text { such that } x_{k^{\prime}}=2, x_{k^{\prime \prime}}=2\right\}
$$

It is easy to prove (exactly the same proof as in the deterministic case) that $U$ is residual and contains equicontinuous points of $\left(A^{\mathbb{Z}}, F\right)$, i.e. $U \subseteq \mathcal{E}$. We show that $\tilde{x}={ }^{\infty} 0^{\infty}$ is not an equicontinuous point of $\left(A^{\mathbb{Z}}, F\right)$. Let $\delta=2^{-k}, k \geq 0$ and consider the point $y \in B_{\delta}=\left[0^{2 k+1}\right]_{-k}$ such that

$$
y_{i}= \begin{cases}x_{i} & \text { if } i \neq k+1 \\ 1 & \text { if } i=k+1\end{cases}
$$

Then $F^{k+1}(\tilde{x})_{0}=\{0,2\} \neq\{1,2\}=F^{k+1}(y)$, which implies that $\tilde{x}$ is not an equicontinuous point and that $\left(A^{\mathbb{Z}}, F\right)$ is almost equicontinuous but not equicontinuous. To conclude, note that $\tilde{x}={ }^{\infty} 0^{\infty} \in F^{-1}(U)$, but since $\tilde{x} \notin U$ we conclude that $F^{-1}(\mathcal{E}) \not \subset \mathcal{E}$.

In CA equicontinuity is strictly related to the presence of blocking words.

Definition 8. Let $\epsilon=2^{-k}, k \geq 0$. A word $w \in A^{2 d+1}$, is a blocking word if

$$
\forall x, y \in[w]_{-d}, \forall n \geq 0, F^{n}(x)_{[-k, k]}=F^{n}(y)_{[-k, k]} .
$$


In particular, equicontinuous points of $\mathrm{CA}$ are characterized by the presence of infinitely many occurrences of blocking words. This strong characterization implies that the set of equicontinuous points of a cellular automaton is either empty or dense (residual). There is no immediate generalization of such property for blocking words of NCA, as shown by the following example. This leaves open the question whether there are NCA whose set of equicontinuous points is non empty and not dense.

Example 3. (NCA with dense set of equicontinuous points) Consider the NCA $\left(A^{\mathbb{Z}}, F\right)$ on the alphabet $A=\{0,1\}$, defined by the following nondeterministic local rule

$$
\forall a, b, c \in A, f(a, b, c)= \begin{cases}\{0,1,2\} & \text { if } b=2 \\ \{b\} & \text { if } c=2 \\ \{c\} & \text { otherwise }\end{cases}
$$

Note that the function $F$ behaves like the shift map on $\{0,1\}^{\mathbb{Z}}$ and that for every $x \in A^{\mathbb{Z}}, F(x) \cap\{0,1\}^{\mathbb{Z}} \neq \emptyset$. On the other end, the symbol 2 does not move and generates all the other symbols. We first show that $\left(A^{\mathbb{Z}}, F\right)$ has a dense set of equicontinuous points. It is easy to see that $\tilde{x}={ }^{\infty} 2^{\infty}$ is an equicontinuous point, since $\forall \epsilon=2^{-k}, k \geq 0$ and for every $\delta \leq \epsilon$

$$
\forall y \in B_{\delta}(\tilde{x}), \forall n>0, F^{n}(y)_{[-k, k]}=F^{n}(\tilde{x})_{[-k, k]}=A^{2 k+1}
$$

In the same way, for every $w \in A^{*}$, all the points in

$$
\left.U_{w}=\left\{x \in A^{\mathbb{Z}}\left|\exists i \in \mathbb{Z}, x_{[} i, i+\right| w \mid-1\right]=w \wedge \forall j \notin[i, i+|w|-1], x_{j}=2\right\}
$$

are equicontinuous. Then the dense set $U=\cup_{w \in A^{*}} U_{w}$ is contained in $\mathcal{E}$.

Now, fix some $\epsilon=2^{-k}, k \geq 0$. For simplicity we consider $k=0$, but what follows can be generalized to larger $k$. By definition, the word $w=2^{2 k+1}=2$ is blocking. We show that there are sequences containing infinite occurrences of $w$ to left and to the right that are not equicontinuous. Consider, for example, the periodic sequence $\tilde{x}={ }^{\infty}(0002)^{\infty}$, such that $\tilde{x}_{[-1,1]}=000$. Since the word 2 cannot generate all the 3 -words on $\{0,1\}$, for $\epsilon=2^{-1}$ and for every $\delta=2^{-d}$ we can build the configuration $y \in B_{\delta}(\tilde{x})$ such that $y_{[d+1, d+3]}=111$ and $y_{i}=x_{i}$ for every $i \notin[d+1, d+3]$. Then, it is easy to see that:

$$
\exists n>0,111 \in F^{n}(y)_{[-1,1]}, \text { while } \forall n \geq 0,111 \notin F^{n}(\tilde{x})_{[-1,1]}
$$

which implies that $\tilde{x}$ is not a point of equicontinuity for $F$.

\section{Sensitivity}

In sensitive dynamical systems small perturbations of an orbit may lead to significantly different trajectories. In some sense, sensitivity is the opposite of equicontinuity and, in fact, the two notions are strictly related: a sensitive dynamical system cannot have points of equicontinuity. The converse is not generally true, although it is for the CA dynamical systems. The question is open for NCA and it is strictly related to the question whether there is a NCA whose set of equicontinuous points is not empty and not dense. 


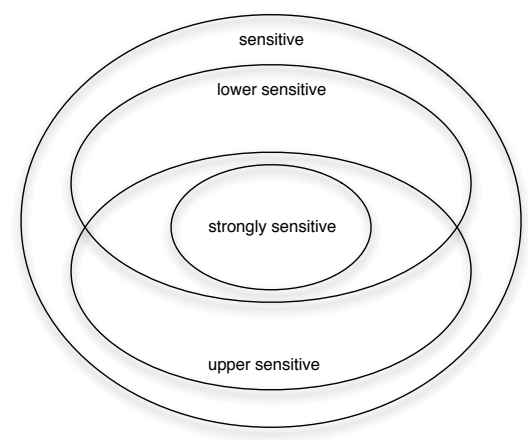

Fig. 3. Sensitivity classes

There is no standard definition of sensitivity for multimaps. We extend the usual definition of sensitivity to multimaps by introducing the notion of upper and lower sensitivity. We get different classes of sensitivity that coincide with the classical definition when the mapping is single-valued.

Definition 9. Let $F: A^{\mathbb{Z}} \rightrightarrows A^{\mathbb{Z}}$ be a $N C A$.

- We say that $\left(A^{\mathbb{Z}}, F\right)$ is upper sensitive (Fig. 1) if

$$
\exists \epsilon>0, \forall x \in A^{\mathbb{Z}}, \forall \delta>0, \exists y \in B_{\delta}(x), \exists n \geq 0, F^{n}(y) \not \subset B_{\epsilon}\left(F^{n}(x)\right)
$$

- We say that $\left(A^{\mathbb{Z}}, F\right)$ is lower sensitive (Fig. 2) if

$$
\exists \epsilon>0, \forall x \in A^{\mathbb{Z}}, \forall \delta>0, \exists y \in B_{\delta}(x), \exists n \geq 0, F^{n}(x) \not \subset B_{\epsilon}\left(F^{n}(y)\right)
$$

- We say that $\left(A^{\mathbb{Z}}, F\right)$ is sensitive if

$$
\begin{gathered}
\exists \epsilon>0, \forall x \in A^{\mathbb{Z}}, \forall \delta>0, \exists y \in B_{\delta}(x), \exists n \geq 0, F^{n}(y) \not \subset B_{\epsilon}\left(F^{n}(x)\right) \vee F^{n}(x) \not \subset \\
B_{\epsilon}\left(F^{n}(y)\right)
\end{gathered}
$$

- We say that $\left(A^{\mathbb{Z}}, F\right)$ is strongly sensitive if

$$
\exists \epsilon>0, \forall x \in A^{\mathbb{Z}}, \forall \delta>0, \exists y \in B_{\delta}(x), \exists n \geq 0, F^{n}(y) \cap B_{\epsilon}\left(F^{n}(x)\right)=\emptyset
$$

The $\epsilon$ constant is called sensitivity constant of the map. All four sensitivity classes imply no equicontinuous points. Note that upper and lower sensitivity imply sensitivity but the converse is not immediately false. Strong sensitivity immediately implies lower and upper sensitivity. While in the deterministic setting all four definitions are equivalent, in the nondeterministic setting, the four definitions give rise to different classes of sensitivity. We show that all such classes are non empty and distinct (see Fig. 3).

In the following two lemmas we prove the following properties: 
1. if some nondeterministic orbit appears in every other orbit, then the NCA is not lower sensitive,

2. if there is a point that is mapped to the entire space, then the NCA is not upper sensitive.

We will use these two properties to build examples of NCA that are not loweror-upper sensitive.

Lemma 1. Let $\left(A^{\mathbb{Z}}, F\right)$ be an NCA. Assume that there is some point $x \in A^{\mathbb{Z}}$ such that

$$
\forall n>0, \forall y \in A^{\mathbb{Z}}, F^{n}(x) \subseteq F^{n}(y)
$$

then $\left(A^{\mathbb{Z}}, F\right)$ is not lower sensitive.

Proof. Let $\left(A^{\mathbb{Z}}, F\right)$ be of radius $r \geq 0$ and assume there is one point $x \in A^{\mathbb{Z}}$ as defined in the statement. Consider some $\epsilon>0$, then

$$
\forall \delta>0, \forall y \in B_{\delta}(x), \forall n>0, F^{n}(x) \subseteq F^{n}(y) \subseteq B_{\epsilon}\left(F^{n}(y)\right),
$$

which implies that $F$ is not lower sensitive.

Lemma 2. Let $\left(A^{\mathbb{Z}}, F\right)$ be an NCA. Assume that there is some point $x \in A^{\mathbb{Z}}$ such that

$$
F(x)=A^{\mathbb{Z}}
$$

Then $F$ in not upper sensitive.

Proof. First of all, note that if $F(x)=A^{\mathbb{Z}}$, then $\forall n>0, F^{n}(x)=A^{\mathbb{Z}}$. Consider some $\epsilon>0$, then

$$
\forall \delta>0, \forall y \in B_{\delta}(x), \forall n>0, F^{n}(y) \subseteq B_{\epsilon}\left(F^{n}(x)\right)=B_{\epsilon}\left(A^{\mathbb{Z}}\right),
$$

which implies that $F$ is not upper sensitive.

All the following examples are based on the shift map. We first show that sensitivity does not imply lower and upper sensitivity.

Example 4. (Sensitive but not lower/upper sensitive NCA) Consider the NCA $\left(A^{\mathbb{Z}}, F\right)$ on alphabet $A=\{0,1\}$ defined by the following nondeterministic local rule:

$$
\forall a, b, c \in A, f(a, b, c)= \begin{cases}\{0\} & \text { if } c=0 \\ \{0,1\} & \text { if } c=1\end{cases}
$$

This nondeterministic map contains both the shift map and the constant map, which sends every configuration to the uniform configuration ${ }^{\infty} 0^{\infty}$.

We first show that $\left(A^{\mathbb{Z}}, F\right)$ is sensitive. Consider some configuration $x \in A^{\mathbb{Z}}$ and note that, by definition of the local rule $f$, for every $i>0$

$$
F^{i}(x)_{0}= \begin{cases}\{0\} & \text { if } x_{i}=0 \\ \{0,1\} & \text { if } x_{i}=1\end{cases}
$$


Let $x \in A^{\mathbb{Z}}$ and let $k>0$. Let $y \in\left[x_{[-k, k]}\right]_{-k}$ be such that

$$
y_{i}= \begin{cases}x_{i} & \text { if } i \neq k+1 \\ 1-x_{i} & \text { if } i=k+1\end{cases}
$$

Then $F^{k+1}(x)_{0} \neq F^{k+1}(y)_{0}$, which implies that $F$ is sensitive with sensitivity constant $\epsilon=2^{0}$. We now show that $F$ is neither lower nor upper sensitive.

1. $F$ is not upper sensitive. Consider the configuration $\tilde{x}={ }^{\infty} 1^{\infty} \in A^{\mathbb{Z}}$. We have that, $\tilde{x}$ is mapped to the entire configuration space, i.e.

$$
\forall n>0, F^{n}(\tilde{x})=A^{\mathbb{Z}} .
$$

then, by Lemma $2, F$ is not upper sensitive.

2. $F$ is not lower sensitive. Consider the configuration $\tilde{x}={ }^{\infty} 0^{\infty} \in A^{\mathbb{Z}}$. We have that $\tilde{x}$ is a quiescent configuration that appears in every orbit, i.e.

$$
\forall n>0, \forall y \in A^{\mathbb{Z}}, F^{n}(\tilde{x})=\{\tilde{x}\} \subseteq F^{n}(y) .
$$

then, by Lemma $1, F$ is not lower sensitive.

The following two examples show that upper sensitivity does not imply lower sensitivity, and conversely.

Example 5. (Upper sensitive and not lower sensitive NCA) Consider the NCA $\left(A^{\mathbb{Z}}, F\right)$ on alphabet $A=\{0,1,2\}$ defined by the following nondeterministic local rule:

$$
\forall a, b, c \in A, f(a, b, c)= \begin{cases}\{0\} & \text { if } c=0 \\ \{0,1\} & \text { if } c=1 \\ \{0,2\} & \text { if } c=2\end{cases}
$$

Consider some configuration $x \in A^{\mathbb{Z}}$ and note that, by definition of the local rule $f$, for every $i>0$

- if $x_{i}=0$, then $F^{i}(x)_{0}=\{0\}$,

- if $x_{i}=1$, then $F^{i}(x)_{0}=\{0,1\}$,

- if $x_{i}=2$, then $F^{i}(x)_{0}=\{0,2\}$,

Then, for every $x \in A^{\mathbb{Z}}$ and $\delta=2^{-k}, k \geq 0$ we can build the configuration $y \in B_{\delta}(x)=\left[x_{[-k, k]}\right]_{-k}$ such that

$$
y_{i}=\left\{\begin{array}{l}
x_{i} \text { if } i \neq k+1 \\
2 \text { if } i=k+1 \text { and } x_{i} \in\{0,1\} \\
1 \text { if } i=k+1 \text { and } x_{i}=2
\end{array}\right.
$$

It is clear that $F^{k+1}(y)_{0} \not \subset F^{k+1}(x)_{0}$, which proves that $F$ is upper sensitive with sensitivity constant $\epsilon=2^{0}$. In order to see that $F$ is not lower sensitive, consider the uniform configuration $\tilde{x}={ }^{\infty} 0^{\infty} \in A^{\mathbb{Z}}$, which is mapped to itself, i.e. $\forall n \geq 0, F^{n}(\tilde{x})=\{\tilde{x}\}$. Note that $\forall y \in A^{\mathbb{Z}}$ and $\forall n>0, F^{n}(\tilde{x}) \subseteq F^{n}(y)$, then by Lemma $1, F$ is not lower sensitive. 
Example 6. (Lower sensitive and not upper sensitive NCA) Consider the one-sided NCA on alphabet $A=\{0,1,2\}$ defined by the following nondeterministic local rule:

$$
\forall a, b, c \in A, f(a, b, c)= \begin{cases}\{0,1,2\} & \text { if } a=b=c=0 \\ \{c\} & \text { otherwise }\end{cases}
$$

Note that, for every $x \in A^{\mathbb{Z}}$ and $i>0$

$$
F^{i}(x)_{0}= \begin{cases}\{0,1,2\} & \text { if } x_{[i-2, i]}=000 \\ \left\{x_{i}\right\} & \text { otherwise }\end{cases}
$$

For every $x \in A^{\mathbb{Z}}$ and for every $\delta=2^{-k}, k \geq 0$ we can build the configuration $y \in B_{\delta}(x)=\left[x_{[-k, k]}\right]_{-k}$ such that

$$
y_{i}=\left\{\begin{array}{l}
x_{i} \text { if } i \neq k+1 \\
2 \text { if } i=k+1 \text { and } x_{i} \in\{0,1\} \\
1 \text { if } i=k+1 \text { and } x_{i}=2
\end{array}\right.
$$

By construction, we have that, if $F^{k+1}(x)=\{0,1,2\}$ or $F^{k+1}(x)=\{1\}$, then $F^{k+1}(y)=\{2\}$, while if $F^{k+1}(x)=\{1\}$ then $F^{k+1}(y)=\{2\}$. In both cases, $F^{k+1}(x) \not \subset F^{k+1}(y)$, which implies that $F$ is lower sensitive with sensitivity constant $\epsilon=2^{0}$. In order to see that $F$ is not upper sensitive, note that the configuration $\tilde{x}={ }^{\infty} 0^{\infty} \in A^{\mathbb{Z}}$ is mapped to the entire space, i.e. $F^{n}(\tilde{x})=A^{\mathbb{Z}}$, $\forall n>0$. Then, by Lemma $2, F$ is not upper sensitive.

Since CA are a subset of NCA, all sensitive CA belong to the strongly sensitive class. We show that such class contains also strictly NCA. The simples example is the nondeterministic reformulation of the shift map.

Example 7. (Strongly sensitive NCA) Consider the NCA $\left(A^{\mathbb{Z}}, F\right)$ on alphabet $A=\{0,1,2.3\}$ defined by the following nondeterministic local rule:

$$
\forall a, b, c \in A, f(a, b, c)=\left\{\begin{array}{l}
\{0,2\} \text { if } c \in\{0,2\} \\
\{1,3\} \text { if } c \in\{1,3\}
\end{array}\right.
$$

Note that, $F$ is essentially a nondeterministic shift map on the two sets $\{0,2\}$ and $\{1,3\}$ :

$$
\forall i>0, F^{i}(x)_{0}=\left\{\begin{array}{l}
\{0,2\} \text { if } x_{i} \in\{0,2\} \\
\{1,3\} \text { if } x_{i} \in\{1,3\}
\end{array}\right.
$$

For every $x \in A^{\mathbb{Z}}$ and $\delta=2^{-k}, k \geq 0$ there is the configuration $y \in B_{\delta}(x)$ such that

$$
y_{i}= \begin{cases}x_{i} & \text { if } i \neq k+1 \\ \left(x_{i}+1\right) \bmod 4 & \text { if } i=k+1\end{cases}
$$

It is easy to see that $F^{k+1}(x)_{0} \cap F^{k+1}(y)_{0}=\emptyset$, which implies that $F$ is strongly sensitive with sensitivity constant $\epsilon=2^{0}$.

It is open the question whether there are NCA, both upper and lower sensitive, that are not strongly sensitive. 


\section{Transitivity}

In a topologically transitive dynamical system every open set has points whose orbits intersect any other open set. While, in general, a transitive dynamical system can be either sensitive or almost equicontinuous, it is well known that topologically transitive CA are sensitive. This strong characteristic does not hold for NCA.

Definition 10. A NCA $\left(A^{\mathbb{Z}}, F\right)$ is topologically transitive if for every nonempty open sets $U, V \in A^{\mathbb{Z}}, \exists n \geq 0, F^{n}(U) \cap V \neq 0$.

The following general property holds for any continuous endomorphism of a compact space.

Proposition 3. Any transitive NCA is surjective.

Proof. Since $F$ is topologically transitive, for every non-empty open set $U \in A^{\mathbb{Z}}$, $F\left(A^{\mathbb{Z}}\right) \cap U \neq \emptyset$, which implies that $F\left(A^{\mathbb{Z}}\right)$ is dense in $A^{\mathbb{Z}}$. Since $F$ is continuous and $A^{\mathbb{Z}}$ compact, $F\left(A^{\mathbb{Z}}\right)$ is closed, then $F\left(A^{\mathbb{Z}}\right)=A^{\mathbb{Z}}$.

The following sufficient condition is useful to build examples of transitive NCA.

Definition 11. Let $\left(A^{\mathbb{Z}}, F\right)$ be a NCA with local rule $f: A^{2 r+1} \rightrightarrows A$. We say that $F^{\prime}$ is a sub-NCA of $F$ if its local rule $f^{\prime}: A^{2 r+1} \rightrightarrows A$ is such that

$$
\forall w \in A^{2 r+1}, f^{\prime}(w) \subseteq f(w) .
$$

If a sub-NCA $F^{\prime}$ of $F$ is deterministic, we denote it as sub-CA.

Lemma 3. If there is a sub-NCA $F^{\prime}$ such that $\left(A^{\mathbb{Z}}, F^{\prime}\right)$ is transitive, then $\left(A^{\mathbb{Z}}, F\right)$ is transitive.

Proof. If $\left(A^{\mathbb{Z}}, F^{\prime}\right)$ is transitive then, for every non-empty open sets $U, V \subseteq A^{\mathbb{Z}}$ there is $n \geq 0$ such that $F^{n}(U) \cap V \supseteq\left(F^{\prime}\right)^{n}(U) \cap V \neq \emptyset$.

By Lemma 3, all the examples of sensitive NCA in Section 4 are transitive, since all of them contain the shift map as sub-CA. However, while it is well known that transitive $\mathrm{CA}$ are sensitive, this is not true in the nondeterministic setting. We conclude this section by showing examples of reversible and not reversible transitive NCA that are equicontinuous.

The most simple example of transitive NCA is the map that sends every point into the entire configuration space. Such map is equicontinuous.

Example 8. (Transitive, reversible and equicontinuous NCA). Consider the NCA on alphabet $A=\{0,1\}$ defined by the following nondeterministic local rule of radius zero

$$
\forall a \in A, f(a)=A
$$

By Proposition $2,\left(A^{\mathbb{Z}}, F\right)$ is equicontinuous. It is clearly transitive, since $\forall n>0$, $\forall x \in A^{\mathbb{Z}}$, and for every open set $U \subseteq A^{\mathbb{Z}}, F^{n}(x) \cap U=A^{\mathbb{Z}} \cap U=U$. This example is also easily reversible and the inverse is the map itself. 
With a small modification of the previous example, we can get a non-reversible, equicontinuous and transitive NCA.

Example 9. (Transitive, irreversible and equicontinuous NCA). Consider the NCA on alphabet $A=\{0,1\}$ defined by the following nondeterministic local rule:

$$
\forall a, b, c \in A, f(a, b, c)= \begin{cases}\{0\} \quad \text { if } a=c=1, b=0 \\ \{0,1\} \text { otherwise }\end{cases}
$$

By Lemma $3,\left(A^{\mathbb{Z}}, F\right)$ is transitive, since it contains the sensitive elementary rule 90. It is easy to see that it is equicontinuous, since $\forall x \in A^{\mathbb{Z}}, \forall n \geq 2, F^{n}(x)=A^{\mathbb{Z}}$. In order to see that it is not reversible, consider the configurations $\tilde{x}={ }^{\infty} 1^{\infty}$ and $\tilde{y}={ }^{\infty} 0^{\infty}$. Note that $\tilde{x} \in F(\tilde{x})$ and $\tilde{x} \in F(\tilde{y})$, thus, if $F^{-1}$ is a nondeterministic block map, the only possibility is that for some $r \geq 0, f^{-1}\left(1^{2 r+1}\right)=\{0,1\}$, which implies that $F^{-1}(\tilde{x})=A^{\mathbb{Z}}$. This is not possible, since $\tilde{x} \notin F\left({ }^{\infty}(01)^{\infty}\right)$.

\section{Conclusions}

We investigated topological dynamical properties of Nondeterministic Cellular Automata. First, we extended to multivalued functions the notions of equicontinuity and sensitivity, which do not have a standard definition as in the singlevalued setting. Then, we studied the classes of equicontinuous, almost equicontinuous and sensitive NCA and their intersections with the class of transitive NCA. The topological dynamics of NCA is extremely complex and there are strong differences with respect to their deterministic counterpart. The largest differences are probably found in the class of equicontinuous NCA. Surjective and equicontinuous CA have a strongly periodic behavior, hence they are bijective and reversible. On the contrary, there are equicontinuous NCA that are transitive and not reversible.

There are several interesting open questions. It is unknown whether there is a NCA whose set of equicontinuous point is not empty and not dense. This question is strictly related to the question whether there are NCA that are not sensitive and not almost equicontinuous, which is also open.

\section{References}

1. Berge, C.: Topological spaces. Including a treatment of multi-valued functions, vector spaces and convexity. Dover Publications, Inc., Mineola, NY (1963).

2. Burkhead, E., Hawkins, J. M.: Nondeterministic and stochastic cellular automata and virus dynamics. J. Cell. Autom. (2016). To appear.

3. Dennunzio, A., Di Lena, P., Formenti, E., Margara, L.: On the directional dynamics of additive cellular automata. Theoret. Comput. Sci. 410, 4823-4833 (2009)

4. Dennunzio, A., Formenti, E., Manzoni, L., Mauri, G.: m-Asynchronous cellular automata: from fairness to quasi-fairness. Natural Computing 12, 561-572 (2013)

5. Dennunzio, A., Formenti, E., Provillard, J.: Local rule distributions, language complexity and non-uniform cellular automata. Theor. Comput. Sci. 504, 38-51 (2013) 
6. Dennunzio, A., Di Lena, P., Formenti, E., Margara, L.: Periodic Orbits and Dynamical Complexity in Cellular Automata. Fundam. Inform. 126, 183-199 (2013)

7. Dennunzio, A., Formenti, E., Weiss, M.: Multidimensional cellular automata: closing property, quasi-expansivity, and (un)decidability issues. Theor. Comput. Sci. 516, 40-59 (2014)

8. Dennunzio, A., Formenti, E., Manzoni, L., Mauri, G., Porreca, A.E.: Computational complexity of finite asynchronous cellular automata. Theor. Comput. Sci. 664, 131-143 (2017)

9. Di Lena, P.: Decidable properties for regular cellular automata, In: Fourth IFIP International Conference on Theoretical computer Science, pp. 185-196 (2006)

10. Di Lena, P. , Margara, L.: Computational complexity of dynamical systems: the case of cellular automata. Inform. Comput. 206, 1104-1116 (2008)

11. Di Lena, P., Margara, L.: On the undecidability of the limit behavior of Cellular Automata. Theoret. Comput. Sci. 411, 1075-1084 (2010)

12. Di Lena, P., Margara, L.: On the Undecidability of Attractor Properties for Cellular Automata. Fund. Inform. 115, 78-85 (2012)

13. Di Lena, P. , Margara, L.: Nondeterministic Cellular Automata. Inform. Sciences 287, 13-25 (2014)

14. Furusawa H., Ishida T., Kawahara Y.: Continuous Relations and Richardson's Theorem. In: Kahl W., Griffin T.G. (eds) Relational and Algebraic Methods in Computer Science. Lecture Notes in Computer Science, vol 7560. Springer, Berlin, Heidelberg (2012)

15. Furusawa, H.: Uniform Continuity of Relations and Nondeterministic Cellular Automata. Theoret. Comput. Sci. In press (2016)

16. Hedlund, G. A.: Endomorphisms and automorphisms of the shift dynamical system. Math. Systems Theory 3, 320-375 (1969)

17. Kůrka, P.: Languages, equicontinuity and attractors in cellular automata. Ergod. Theor. Dyn. Syst. 17, 417-433 (1997)

18. Kůrka, P. : Topological and Symbolic Dynamics. Cours Spécialisés 11, Société Mathématique de France, Paris (2003)

19. Ozhigov, Y. : Computations on Nondeterministic Cellular Automata. Inform. Comput. 148, 181-201 (1999).

20. Richardson, D. : Tessellations with local transformations. J. Comput. Syst. Sci. 6, 373-388 (1972)

21. Yaku, T.: Surjectivity of Nondeterministic Parallel Maps Induced by Nondeterministic Cellular Automata. J. Comput. Syst. Sci. 12, 1-5 (1976) 